\title{
Heterogeneity of Hepatocellular Carcinoma on Imaging
}

\author{
Jordi Rimola, MD, PhD ${ }^{1 \odot}$ \\ ${ }^{1}$ Department of Radiology, BCLC group, Hospital Clínic Barcelona, \\ CIBER-ehd, University of Barcelona, Barcelona, Catalonia, Spain \\ Semin Liver Dis 2020;40:61-69.
}

\begin{abstract}
Address for correspondence Jordi Rimola, MD PhD, Department of Radiology, BCLC group, Hospital Clínic Barcelona, CIBER-ehd, University of Barcelona, C/Villarroel 170, Escala 3 Planta 1, Barcelona, 08036, Catalonia, Spain (e-mail: jrimola@clinic.cat).
\end{abstract}

\author{
Abstract \\ Keywords \\ - hepatocellular \\ carcinoma \\ - liver \\ - magnetic resonance \\ imaging \\ - contrast media \\ - heterogeneity
}

Tumor heterogeneity in hepatocellular carcinomas (HCC) occurs at different levels. On conventional imaging modalities, the spectrum of tumor heterogeneity is evident in the dynamic enhancement pattern, where the characteristic wash-in and wash-out is present in only $60 \%$ of small HCCs. In larger HCCs, heterogeneity within the tumor, known as the mosaic pattern, can reflect the presence of different grades of HCC differentiation. The advent of functional imaging techniques has not improved the diagnostic sensitivity of imaging techniques for the diagnosis of HCC. However, the combination of conventional and functional imaging techniques potentially allows the identification of heterogeneity in tumor vascularity, cellularity, and molecular expression.
The noninvasive diagnosis of hepatocellular carcinoma (HCC) represents a major challenge for radiologists. In cirrhotic livers, a new focal lesion has a high pre-test probability of being an HCC. $^{1-3}$

Magnetic resonance imaging (MRI) has a key role in detecting and characterizing HCCs in cirrhotic livers., ${ }^{4,5}$ MRI has superior tissue resolution compared with computed tomography (CT). Different meta-analysis and systemic reviews comparing the diagnostic performance of contrastenhanced CT in comparison to contrast-enhanced MRI found that multiphasic MRI is more sensitive than CT, while both modalities have similar specificity. ${ }^{6,7}$ MRI contrast agents improve detection by increasing the contrast between the lesion and the background, ${ }^{4,6}$ and different types of contrast agents can improve characterization by showing changes in hepatocyte uptake; biliary excretion; or vascular, extracellular, or intracellular volumes. ${ }^{6,8}$ This information can be complemented by information obtained from other sequences such as restriction on DWI, high signal on T2-weighted images, and presence of intralesional fat. Nevertheless, despite considerable recent advances in MRI, the final diagnosis of new focal lesions often relies on histopathology. 4

Biopsies are required for diagnosis or staging when imaging techniques cannot characterize new liver lesions in cirrhotic patients. Histopathology is still needed for two main reasons: first, HCC is heterogeneous on imaging, with different lesions within the same patient and even the same tumor having different appearances. Second, HCC is the endstage of a continuous multistep process, ${ }^{9}$ and the MRI findings for pre-malignant lesions and early HCC are not specific and overlap. ${ }^{10}$ Hence, the main question is whether a given nodule is a large regenerative nodule, a dysplastic nodule, or an HCC. The aim is to avoid overdiagnosis and consequent overtreatment while avoiding underdiagnosis and consequent undertreatment.

\section{Heterogeneity Using Conventional Imaging Criteria}

Hepatocarcinogenesis is a multistep process during which nodules undergo parallel, simultaneous, and progressive changes, including increased cell density, decreased cell differentiation, enlargement of nodules, and changes in nodule hemodynamics. ${ }^{9}$ The MRI characterization of focal lesions in cirrhotic livers is a complex process based on analyzing the lesion's signal intensity on different imaging sequences. In dynamic contrast-enhanced MRI, the signal intensity during the arterial, portal venous, and delayed venous phases reflects differences in the distribution of contrast material between the vascular and extravascular spaces in tumors and liver parenchyma; the noninvasive diagnostic criteria for HCC are based on these characteristics. ${ }^{4,5,11}$ published online

July 2, 2019
Copyright (c) 2020 by Thieme Medical Publishers, Inc., 333 Seventh Avenue, New York, NY 10001, USA. Tel: +1(212) 760-0888.
DOI https://doi.org/ 10.1055/s-0039-1693512. ISSN 0272-8087. 
In the initial phase of hepatocarcinogenesis, normal arterial supply decreases but portal venous supply is still present. $^{10,12,13}$ In a more advanced stage, intranodular arterial vascularity increases due to the appearance of unpaired feeding arteries while venous portal blood supply progressively decreases. These phenomena result in the typical and specific enhancement pattern of HCC on MRI using nonspecific gadolinium contrast: arterial hypervascularity and hypointensity or "washout" during the portal venous and/ or late dynamic phase. These features represent the European Association for the Study of the Liver (EASL) and the American Association for the Study of Liver Diseases (AASLD) noninvasive diagnostic criteria for HCC larger than $1 \mathrm{~cm}$. Although this pattern is highly specific, it has limited sensitivity, being found in only $\sim 60 \%$ of HCC smaller than $2 \mathrm{~cm},{ }^{1-3,14,15}$ because early small HCC in which neoangiogenesis is yet to progress is often hypovascular. The difficulties in differentiating the different stages of HCC progression by imaging, particularly in differentiating low-grade dysplastic nodules from regenerative nodules and high-grade dysplastic nodules from early HCC, probably derive from the fact that the different classifications simply represent parts of a continuum rather than distinct categories ( - Fig. $\mathbf{1}){ }^{9}$

Another source of heterogeneity in the appearance of small HCCs is the presence of fat, also known as fatty metamorphosis. Whereas fat is often present in small, well-differentiated HCCs, small moderately-to-poorly differentiated HCCs rarely contain fat. $^{2}$ Moreover, fatty metamorphosis can also be found in dysplastic nodules and benign entities. Vascular changes sometimes appear in small areas inside a dysplastic nodule or an early HCC, conferring the classical appearance of a nodule within a nodule.

As HCCs grow larger, the heterogeneity of their appearance decreases as the typical vascular pattern develops; this pattern can be identified in more than 70\% of HCC between 2 and $3 \mathrm{~cm}$ in diameter. ${ }^{6,7,16}$ At the same time, heterogeneity within lesions increases as different foci of HCC differentiation coexist, conferring a mosaic pattern resulting from areas with different degrees of vascularization, fatty metamorphosis, and/or hemorrhage. ${ }^{17}$

In 7 to $13 \%$ of cases, HCC presents as the diffuse or infiltrative HCC subtype, ${ }^{18}$ which is often accompanied by portal vein thrombosis, resulting in vascular phenomena and abnormal patterns of enhancement. Infiltrative HCC is easily missed on T1-weighted images and on arterial phase images, where they usually show no enhancement or slight heterogeneous enhancement within the tumor and the large heterogeneous tumor with ill-defined margins blends into the heterogeneous cirrhotic liver. ${ }^{19}$

Another source of heterogeneity by imaging are the primary liver carcinomas with both hepatocyte and cholangiocyte differentiation, referred to as "combined (or mixed) hepatocellular-cholangiocarcinoma." Recently, these tumors have increased their attention because of greater frequency and clinical recognition. ${ }^{20}$ There are few publications that

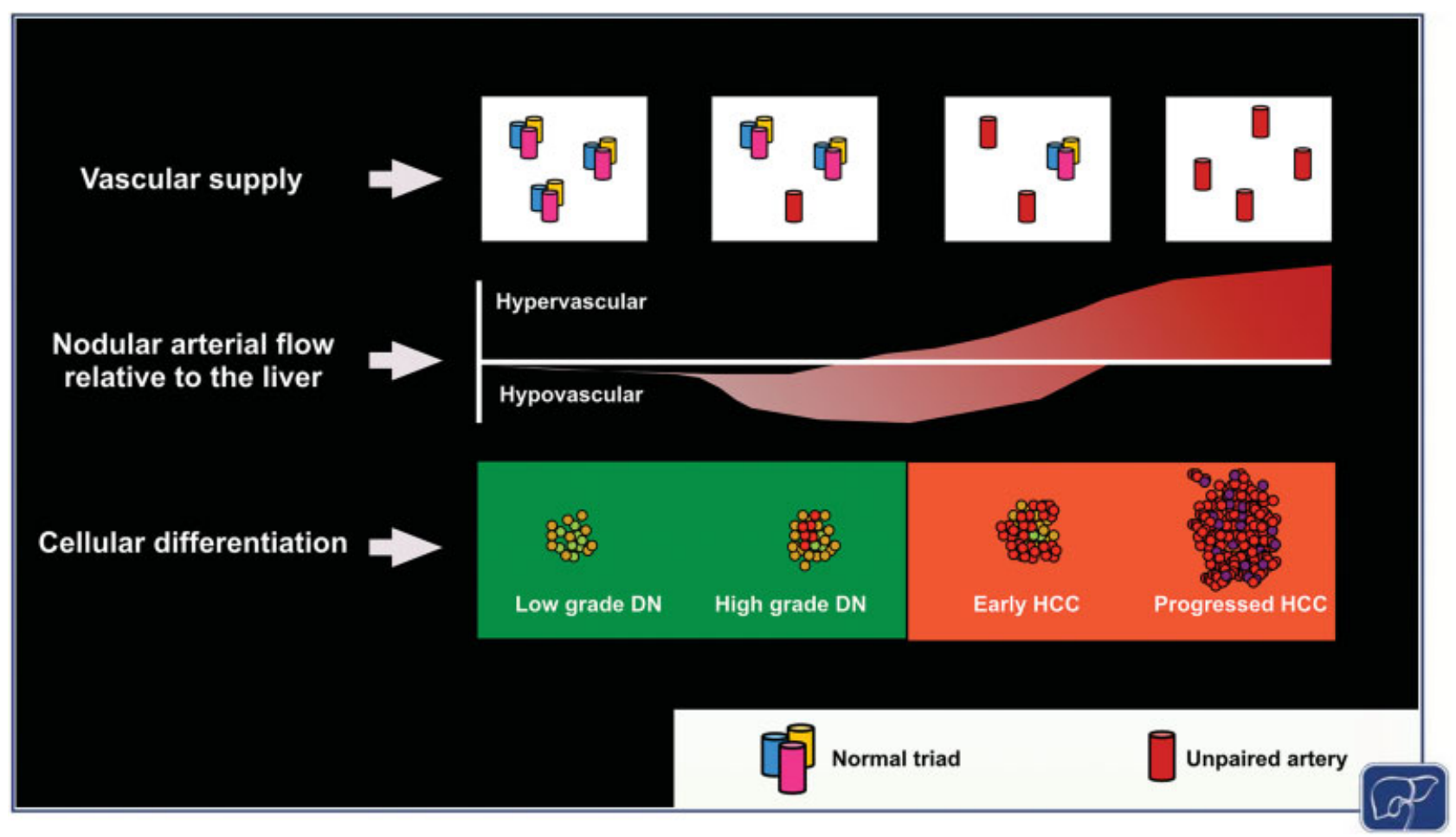

Fig. 1 Phenotypic changes along the continuous process of hepatocarcinogenesis that cause heterogeneity at imaging are marked by the following phases at the histologic level: (1) low- and high-grade dysplastic nodules, which on magnetic resonance imaging (MRI) usually appear iso- or hypovascularized (although a few are hypervascular) compared with liver parenchyma, which histology shows have individual arteries and portal perfusion; (2) early hepatocellular carcinoma (HCC) with residual portal perfusion, which on MRI ranges from iso- or hypo-vascular to hypervascular, which histology shows are well differentiated, vaguely nodular, and contain individual arteries; and (3) progressed HCC, with characteristic imaging features of hypervascularity in the arterial phase and hypovascularity in the venous phase, which histology shows are moderately differentiated, distinctly nodular, with or without vascular invasion, having individual arteries but no portal tracts. 
report the radiological appearances of mixed hepatocellularcholangiocarcinoma. Most series reported imaging features that commonly overlap with those of intrahepatic cholangiocarcinoma and less frequently with those of classic HCC displaying predominantly arterial hyperenhancement and followed by diffuse or patchy washout. ${ }^{20}$ Imaging techniques may be useful to guide biopsy on the target HCC-like or cholangiocarcinoma-like areas.

\section{Heterogeneity on Functional Imaging Techniques}

\section{Diffusion-Weighted Imaging}

Diffusion-weighted imaging (DWI) is a functional MRI sequence that allows the characterization of biological tissues based on the diffusion properties of water molecules. This sequence provides indirect information about cell density and about the integrity of cell membranes in the area of interest. In clinical practice, the concept of "diffusion restriction" refers to a focal liver lesion showing signal intensity higher than the surrounding liver parenchyma on DWI. ${ }^{21}$

Diffusion-weighted imaging now forms part of most routine liver MRI protocols in daily practice because it has a short acquisition time, does not require intravenous contrast agents, and is more sensitive for detecting focal liver lesions than T2weighted sequences. ${ }^{22}$ Although HCCs typically appear hyperintense at DWI, this finding is not specific for HCC; thus, DWI should be integrated in MRI protocols together with dynamic sequences, rather than used alone (- Fig. 2) ${ }^{23-25}$ DWI's sensitivity for detecting HCC in cirrhotic livers varies widely depending on the size of the tumor and DWI specifications, ranging from 32 to $70 \%$ in HCC smaller than $2 \mathrm{~cm}^{22-24,26-28}$ to more than $90 \%$ in larger lesions. ${ }^{29,30}$

The heterogeneity in the visibility and signal intensity of HCC at DWI is the product of complex interactions between various biological factors and MRI characteristics. As the process of hepatocarcinogenesis and tumor differentiation occur in a continuum, the heterogeneity of HCCs also results in overlapping diffusivity characteristics that directly affect DWI's ability to detect these lesions. Nasu et $\mathrm{al}^{30}$ reported that the DWI signal intensity of HCC generally increased with increasing histologic grade (i.e., decreasing differentiation), although with large overlaps. Analyzing a series of 98 lesions in 73 patients, Muhi et $\mathrm{al}^{28}$ found that more than $90 \%$ of moderately-to-poorly differentiated HCCs were
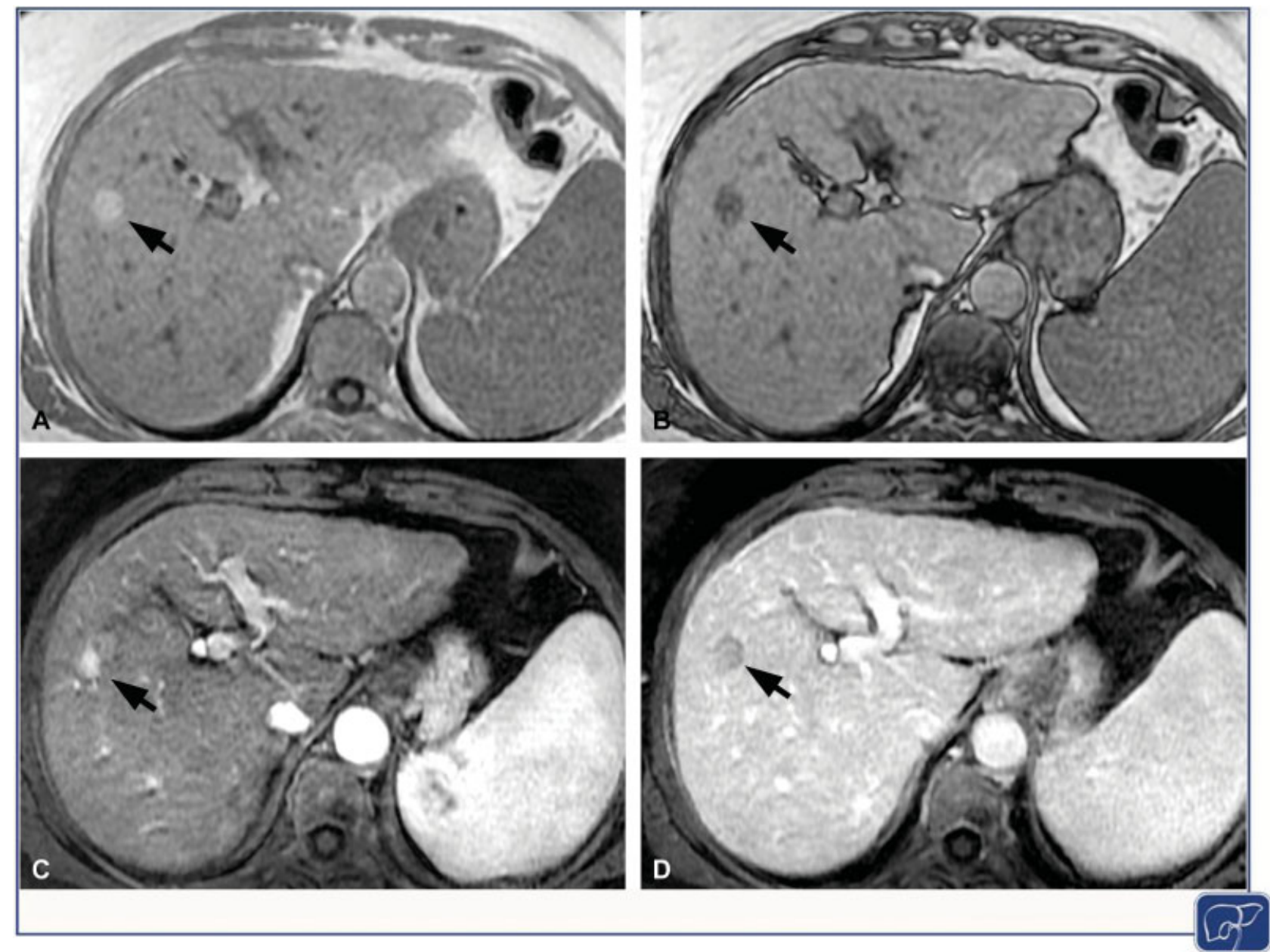

Fig. 2 A $1.2 \mathrm{~cm}$ hepatocellular carcinoma located in segment VIII seen on T1 pre-contrast in (A) and opposite phase (B), arterial phase (C), and portal venous phase (D) magnetic resonance imaging. On pre-contrast T1-weighted images, the nodule presented drop in signal on opposite phase relative to liver parenchyma (arrow in A and B) indicating presence of fat. On arterial-phase images, hyperenhancement is seen within the nodule (arrow in C). On portal-venous-phase images, the nodule is hypointense (washout) (arrow in D). This vascular pattern is specific for hepatocellular carcinoma. 
hyperintense on DWI, but only $51 \%$ of well-differentiated HCCs and none of the dysplastic nodules were hyperintense. Moreover, the microarchitecture of the background cirrhotic parenchyma also influences the visibility of HCCs on DWI, increasing the complexity of interpreting the findings. Diffusivity is also restricted in cirrhotic parenchyma, probably due to fibrosis, so the DWI signal ratio between HCC and cirrhotic parenchyma is reduced. ${ }^{31}$ This may explain why the DWI detection rate for HCC decreases with increasing severity of cirrhosis. ${ }^{30}$

Although qualitative visual assessment of DWI is of limited usefulness in characterizing nodules in cirrhotic liver, quantitative approaches such as apparent diffusion coefficients (ADC) and the more recent intravoxel incoherent motion (IVIM) can assess nodules independent of cirrhotic liver background, displaying the findings in parametric maps. IVIM is more reliable; unlike ADC, which ignores the effect of the perfusion fraction in tissue, IVIM treats DWI signal attenuation as a mixture of tissue perfusion and tissue diffusivity effects. ${ }^{32}$ In a recent study, Zhu et $\mathrm{al}^{32}$ found a negative correlation $(r=-0.62, p<0.001)$ between IVIM quantification and the histological grade of HCCs. In a similar study, Wei et $\mathrm{al}^{33}$ found that the correlation was even better when IVIM was measured in the whole HCC volume $(r=-0.90, p<0.001)$ rather than in a single section $(r=-0.84, p<0.001)$.

\section{Heterogeneity on Liver-Specific Contrast Agents}

Liver-specific (also called hepatobiliary) contrast agents include gadobenate dimeglumine (Gd-BOPTA) and gadoxetic acid (Gd-EOB-DTPA); the latter is by far the most commonly used liver-specific contrast agent in clinical practice and research. Gadoxetic acid's dual behavior is especially useful in liver imaging. After intravenous injection, it first distributes into the intravascular and extravascular spaces during the arterial, portal, and late venous phases; afterward, during the so-called hepatobiliary phase, it progressively distributes into the hepatocytes and bile ducts.

Gadoxetic acid is eliminated through both renal and hepatic excretion pathways in a proportion of around 50\% each in healthy subjects. The uptake of gadoxetic acid by hepatocytes mainly occurs via the active transporter organic anion transporting polypeptides (OATP) 1B1 and OATPB3 located at the sinusoidal pole of the hepatocytes, and the biliary excretion occurs via multidrug resistance protein (MRP) 2 proteins located in the canalicular membrane. ${ }^{34}$ The expression of OATP transporters diminishes during hepatocarcinogenesis ${ }^{35,36}$; consequently, most HCCs appear hypointense during the hepatobiliary phase because the expression of OATP is decreased or absent (-Fig. 3). The uptake pattern of gadoxetic acid by HCC in the hepatobiliary phase can be explained by the expression of OATP and MRP2. When OATP expression is maintained, the intensity of HCC on hepatobiliary-phase images depends on the expression of MRP2 in the canalicular membrane. In most HCCs, MRP2 is present, and the gadoxetic acid is eliminated to the bile ducts, making these HCCs hypointense on MRI ${ }^{36}$ (-Fig. 4).
OATP expression decreases prior to complete neoangiogenesis and the presence of unpaired hepatic arteries. Consequently, nodules that are hypointense in the hepatobiliary phase but do not have the features of HCC in earlier postcontrast phases due to a lack of unpaired arteries are common and may represent high-grade dysplastic nodules or early HCCs. ${ }^{37,38}$

On the other hand, around $10 \%$ of HCCs are hyperintense in the hepatobiliary phase. This contrast uptake is most commonly associated with well-differentiated HCCs and with either pseudoglandular architecture (classically known as green HCCs) or low expression of MPR2 transporters. ${ }^{39,40}$

Although it has been postulated that hepatobiliary-phase hypointensity generally increases with increasing HCC grade, ${ }^{41}$ the reality is more complex, because both tumor and liver characteristics can affect gadoxetic acid uptake and the relative signal intensity of HCC. ${ }^{42}$

Patients with significant liver dysfunction due to the cirrhosis may have decreased parenchymal uptake of gadoxetic acid, reducing the signal contrast between HCC and the surrounding liver; this phenomenon is particularly problematic for the characterization of small tumors. Recently, other biochemical and clinical factors such as prothrombin activity, total bilirubin, and cholesterol levels have been correlated to the quality of liver enhancement in the hepatobiliary phase, suggesting that better enhancement may be obtained in patients with HBV than in those with HCV. ${ }^{43,44}$

\section{Heterogeneity on Liver Perfusion}

Perfusion imaging of the liver with dynamic contrast-enhanced MRI characterizes and quantifies functional microenvironmental changes in small vessels that are beyond the resolution of morphological imaging for direct visualization. Dynamic contrast-enhanced perfusion MRI is based on temporal changes in tissue enhancement after intravenous administration of contrast media ${ }^{45}$ and reflects pathophysiological changes in the microcirculation. Some groups have reported that dynamic contrast-enhanced MRI can quantify perfusion in HCC and surrounding cirrhotic liver, finding increased arterial perfusion and decreased portal venous flow in HCC compared with cirrhotic liver. ${ }^{46,47}$ However, in large HCCs, intratumoral heterogeneity, in particular in histological grade and microvessel density, often hinders characterization by dynamic contrast-enhanced MRI and makes it difficult to correlate the quantitative findings with the histological grade. ${ }^{48}$

\section{Heterogeneity in Radiological Response and Tumor Progression}

Antiangiogenic drugs are recommended as the first treatment option in cirrhotic patients with advanced HCC. The radiological response to systemic treatment of HCC is usually measured by Response Evaluation Criteria in Solid Tumours (RECIST) 1.1 criteria. $^{49}$

After treatment with antiangiogenic drugs, enhancement decreases either diffusely or in part of the tumor in some HCCs, but remains stable in others. As initially reported in the SHARP trial, the reduction in tumor burden measured by conventional RECIST 1.1 does not parallel the benefit of antiangiogenic drugs. Thus, a panel of experts developed a set of guidelines 


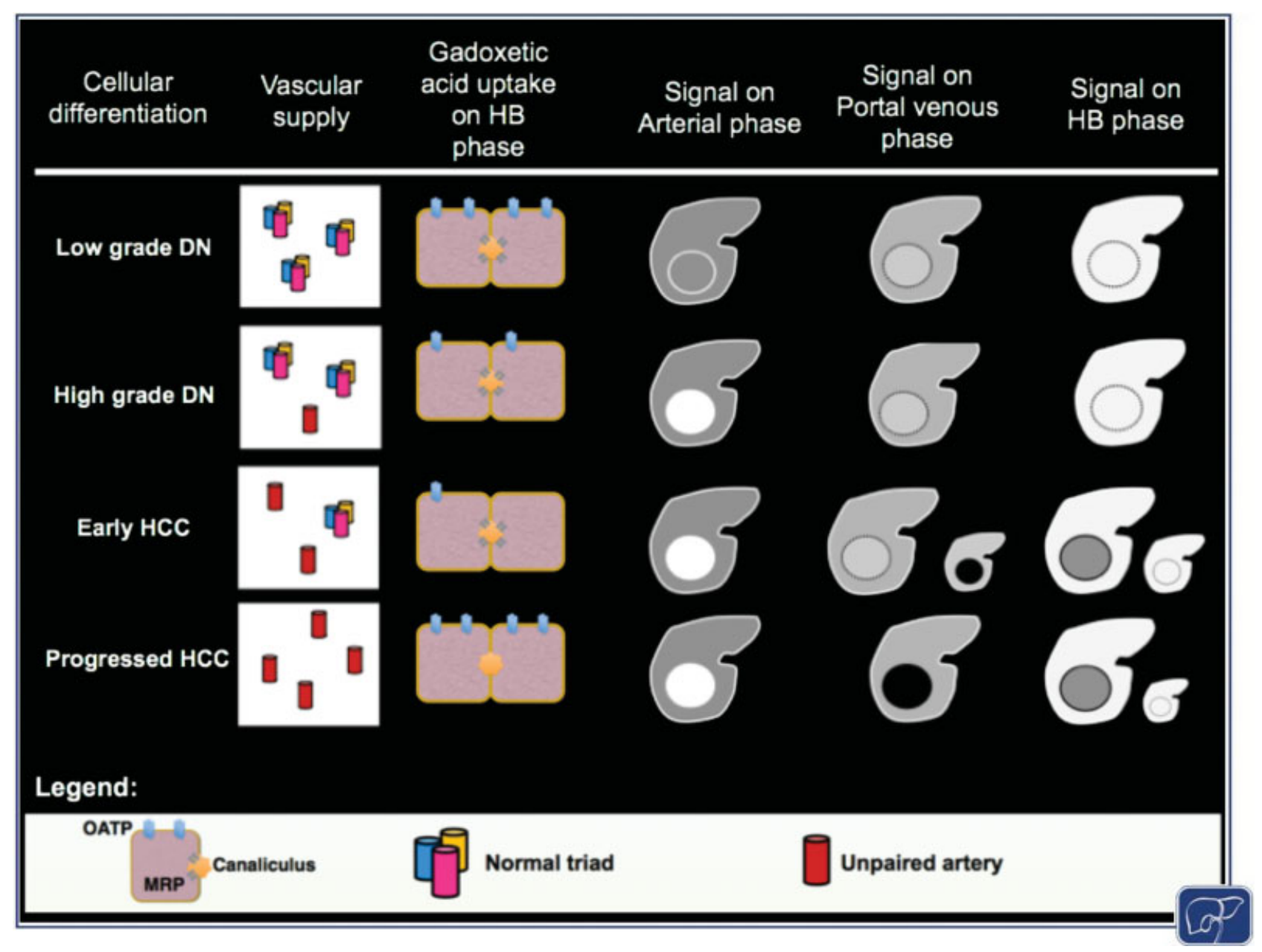

Fig. 3 Correlation between the continuous process of hepatocarcinogenesis and the level of heterogeneity at imaging obtained by liver-specific contrast agents: (1) low- and high-grade dysplastic nodules still have large number of organic anion transporting polypeptides (OATP) and multidrug resistance protein (MRP) transporters that regulate the uptake and excretion of gadoxetic acid, so these nodules usually appear isointense relative to liver parenchyma on hepatobiliary-phase images, although some high-grade dysplastic nodules may appear hypointense. In early and progressed hepatocellular carcinomas, OATP expression is often decreased or absent, resulting in decreased uptake, and MRP expression is often increased, resulting in increased excretion. Thus, most hepatocellular carcinomas appear hypointense during the hepatobiliary phase. However, around 10\% of HCC have increased expression of OATP and decreased expression of MRP, or a pseudogland configuration, both of which result in hepatocellular carcinoma appearing hyperintense on hepatobiliary phase images.

including alternative criteria that take antiangiogenic drugs' mechanism of action into consideration, the modified RECIST for HCC (mRECIST), ${ }^{50}$ thus potentially capturing the heterogeneity on HCC in the response to antiangiogenic drugs beyond mere changes in size. In the BRISK trial, a randomized phase III trial comparing brivanib versus placebo in advanced HCC, Lencioni et $\mathrm{al}^{51}$ analyzed the objective response by mRECIST as a predictor of overall survival in a time-dependent covariate analysis and found a strong association between log hazard ratios for overall survival and log odds ratios for objective response ratio $(r=-0.80 ; 95 \%$ confidence interval: $-1-0.23$, $p=0.091$ ). Note that the $95 \%$ confidence interval included 0 , indicating that the results are not robust enough to assume that the objective response according to mRECIST predicts overall survival in advanced HCC or to consider it a surrogate end-point.

Other authors have compared the rate of objective response using RECIST 1.1 versus mRECIST. Ronot et $\mathrm{al}^{52}$ reported that of a total of 64 patients with HCC treated by sorafenib, only 2 (3\%) had an objective response according to RECIST 1.1, whereas 18 (28\%) had an objective response according to mRECIST. In a similar study, Gavanier et $\mathrm{al}^{53}$ found that only $3 \%$ had an objective response to sorafenib according to RECIST 1.1, whereas $7 \%$ had an objective response to sorafenib according to mRECIST. However, neither study found significant differences in overall survival between patients with an objective response and those with stable disease using either criteria. Interestingly, in both studies the rate of disease progression was very similar using either RECIST 1.1 or mRECIST. The RECIST 1.1 definition of progression takes into account heterogeneity in different situations. Since the response to antiangiogenic treatment is associated with overall survival and helps identify patients who would potentially benefit from second-line therapy, defining the different patterns of response and their implications is important. The BCLC group defined four patterns of radiological progression: intrahepatic increase $\geq 20 \%$ increase in tumor size from the baseline measurement, extrahepatic increase $\geq 20 \%$ increase in tumor 


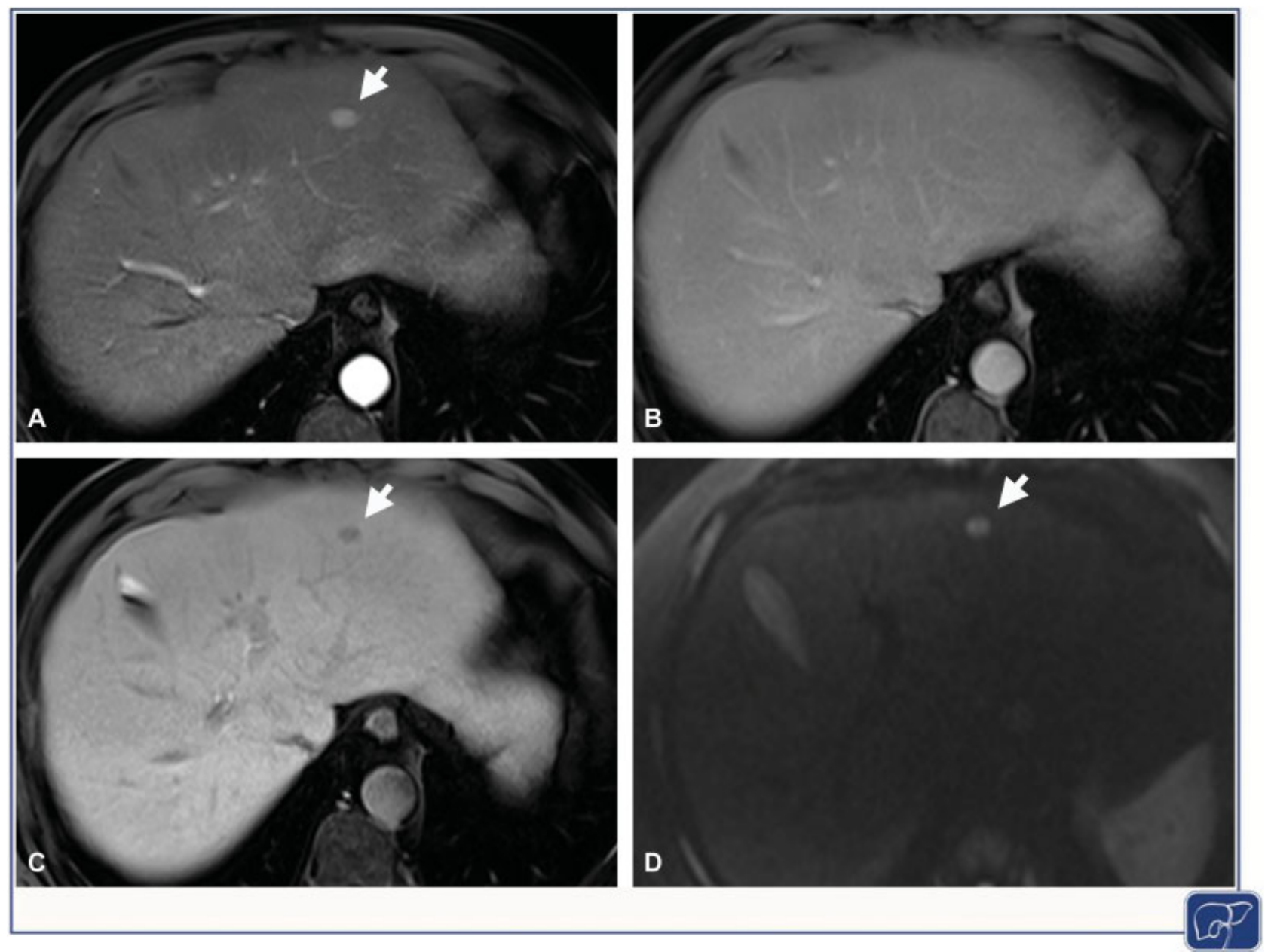

Fig. 4 Gadoxetic-acid liver magnetic resonance imaging (MRI) in a 67-year-old man with hepatitis $C$ virus-related cirrhosis. Liver MRI in the arterial phase shows a $11 \mathrm{~mm}$ hypervascular nodule in segment II of the liver (arrow in A); there was no washout in the portal venous phase (B), and the lesion was hypointense in the hepatobiliary phase (arrow in C). Diffusion-weighted imaging (arrow in D) shows signal restriction. Histopathology after ultrasound-guided biopsy diagnosed well-differentiated hepatocellular carcinoma.

size from the baseline measurement, detection of new intrahepatic lesions, detection of new extrahepatic lesions, and/or vascular invasion. Each pattern is associated with different post-progression survival; intrahepatic growth is the pattern associated with the least aggressive progression, whereas new extrahepatic lesion and/or vascular invasion is associated with the most aggressive progression. ${ }^{54,55}$ This heterogeneity might be explained by molecular factors or gene expression and it deserves further investigation.

Immune modulators are among the most important classes of new anticancer therapeutics. Although their role in systemic treatment for HCC is yet to be determined, various ongoing clinical trials are evaluating their efficacy. The novel mechanism of action of immune checkpoint inhibitors through immune and $\mathrm{T}$ cell activation can result in unusual patterns of response. Some patients treated with this approach showed morphological changes in lesions that would meet the criteria for disease progression based on traditional RECIST (either increase in size or appearance of new lesions), but later showed profound and durable responses. Dubbed pseudoprogression, the increase in the size of lesions after treatment with immune modulators is the result of an inflammatory-like reaction due to an increase in the number of immune cells between tumor cells. $^{56}$

Although pseudoprogression is relatively uncommon, it can be challenging to differentiate this transient phenomenon from true progression that would potentially require a change in the therapeutic approach. Thus, it is essential to know how patients are being treated and the mechanisms of action of these treatments to ensure accurate interpretation of morphological changes observed at MRI or CT and optimal decisions about further workup. In patients receiving immune-checkpoint inhibitors, HCC should be reassessed earlier than originally planned (but only between 4 and 8 weeks after the unconfirmed progression) to determine whether the findings represent real progression or an early tumor response. ${ }^{56,57}$

\section{Future Perspectives}

The noninvasive diagnosis of liver nodules by CT and MRI is limited by the high level of heterogeneity in small HCCs, by the overlapping findings between HCCs and dysplastic nodules, and by atypical vascular patterns. Another potential limitation of the noninvasive diagnosis is the inherent 
difficulties in detecting small nodules during ultrasound surveillance caused by cirrhotic liver background. A recent controversial point is whether the strategy of HCC surveillance should be done by US or by other contrast-enhanced imaging modalities. Surveillance by CT or MR is generally not recommended because of the radiation risks, the higher cost, and the increase in false positive signals due to nonspecific findings. ${ }^{4,5}$

In the last years, few studies have postulated to use MRI with liver-specific contrast agent for screening of HCC in patients with high risk. Authors found that MRI yielded a detection rate of $84.8 \%$, significantly higher than the $27.3 \%$ rate detected by routine US. ${ }^{58}$ The same group published another similar study detecting that screening of HCC using MRI is also cost-effective. ${ }^{59}$ However, there are some aspects of these studies that are at least controversial and may limit the generalization of reported data including (1) the lower rate of US detection reported by this group (27.3\% compared with the $63 \%$ of HCC rate detection of HCC at early stage reported by meta-analysis ${ }^{60}$ ); (2) the high rate of HCC detection at screening MRI (without histological confirmation) suggesting that this strategy may induce HCC over-diagnosis and, in consequence, overtreatment of non-malignant lesions; and (3) concerns on the concept and model for identification of high-risk cirrhotic patients, and the potential bias in the selection of eastern population that can be different than in western countries.

These current limitations of imaging to diagnose HCC limitations have not been overcome with the introduction of functional imaging modalities such as DWI and hepatobiliary phase gadoxetic-acid imaging, which are also affected by heterogeneity and are thus potentially an additional source of diagnostic error.

The classic conceptualization of medical images as pictures intended solely for visual interpretation is an obstacle that must be overcome to improve diagnosis.

Radiomics, which is an emerging field of oncological research, assume that radiologic image features could predict the prognosis of oncologic patients, as they are associated with tumor biological characteristics. In the field of HCC, radiomics had been mainly explored to link image texture features with clinical outcome, by building models that positively correlate with aggressive tumor phenotypes (future fast increase in size and vascular invasion) and with increase in recurrence rate and lower survival.

In that regards, some preliminary studies found that the analysis of texture features on dynamic phases (either arterial or hepatobiliary phases) may help to detect those HCC with higher potential aggressive evolution at baseline (i.e., differentiation low- from high-grade $\mathrm{HCC},{ }^{61}$ to predict the risk of progression from hypovascular nodules into HCC, or to predict risk of recurrence ${ }^{62}$ ) or to predict survival assessed with texture analysis of pre-treatment CT scans. ${ }^{63}$

The analysis of these data can be used to develop decisionsupport tools for diagnosis and to devise predictive or prognostic models for outcomes of interest. ${ }^{64}$ Potentially, these models may complement current existing staging systems such as BCLC and would help to plan a more personalized therapeu- tic option after the initial diagnosis of solitary HCC in cirrhotic patients. However, a common limitation of radiomics studies is the inclusion of radiologic scans acquired in one or few machines. Therefore, expanding the analysis at large multicentric scales might have affected the radiomics results. Further studies and validations using different scanners, models, and contrast agents are required before their application in clinical research or practice.

\section{Main Concepts and Learning Points}

- The aim of this review is to offer a comprehensive view of the heterogeneity of hepatocellular carcinomas at diagnosis on both morphological and functional imaging techniques.

- The noninvasive diagnosis of hepatocellular carcinoma represents a major challenge for radiologists. Hepatocellular carcinomas can be radiologically and histologically heterogeneous within tumors, between tumors, and between patients.

- The spectrum of heterogeneity depends mainly on hepatocellular carcinoma's degree of differentiation and its size. This heterogeneity reflects and correlates with the histologic continuum spanning the process of hepatocarcinogenesis.

- After systemic treatment, there is also heterogeneity in the pattern of changes seen among different hepatocellular carcinomas and in the type of tumor progression. Further research at the molecular and genetic levels is needed to better understand these changes.

Conflicts of Interest

Dr. Rimola reports personal fees from Bayer, outside the submitted work.

\section{References}

1 Forner A, Vilana R, Ayuso C, et al. Diagnosis of hepatic nodules $20 \mathrm{~mm}$ or smaller in cirrhosis: prospective validation of the noninvasive diagnostic criteria for hepatocellular carcinoma. Hepatology 2008;47(01):97-104

2 Rimola J, Forner A, Tremosini S, et al. Non-invasive diagnosis of hepatocellular carcinoma $\leq 2 \mathrm{~cm}$ in cirrhosis. Diagnostic accuracy assessing fat, capsule and signal intensity at dynamic MRI.J Hepatol 2012;56(06):1317-1323

3 Iavarone M, Sangiovanni A, Forzenigo LV, et al. Diagnosis of hepatocellular carcinoma in cirrhosis by dynamic contrast imaging: the importance of tumor cell differentiation. Hepatology 2010;52(05):1723-1730

4 Galle PR, Forner A, Llovet JM, et al; European Association for the Study of the Liver. Electronic address: easloffice@easloffice.eu; European Association for the Study of the Liver. EASL clinical practice guidelines: management of hepatocellular carcinoma. J Hepatol 2018;69(01):182-236

5 Marrero JA, Kulik LM, Sirlin CB, et al. Diagnosis, staging, and management of hepatocellular carcinoma: 2018 Practice Guidance by the American Association for the Study of Liver Diseases. Hepatology 2018;68(02):723-750

6 Roberts LR, Sirlin CB, Zaiem F, et al. Imaging for the diagnosis of hepatocellular carcinoma: a systematic review and meta-analysis. Hepatology 2018;67(01):401-421

7 Lee YJ, Lee JM, Lee JS, et al. Hepatocellular carcinoma: diagnostic performance of multidetector CT and MR imaging-a systematic review and meta-analysis. Radiology 2015;275(01):97-109 
8 Bashir MR. Magnetic resonance contrast agents for liver imaging. Magn Reson Imaging Clin N Am 2014;22(03):283-293

9 Kojiro M, Wanless IR, Alves V, et al; International Consensus Group for Hepatocellular NeoplasiaThe International Consensus Group for Hepatocellular Neoplasia. Pathologic diagnosis of early hepatocellular carcinoma: a report of the international consensus group for hepatocellular neoplasia. Hepatology 2009;49(02):658-664

10 Kudo M. Multistep human hepatocarcinogenesis: correlation of imaging with pathology.J Gastroenterol 2009;44(Suppl 19):112-118

11 Chernyak V, Fowler KJ, Kamaya A, et al. Liver Imaging Reporting and Data System (LI-RADS) Version 2018: imaging of hepatocellular carcinoma in at-risk patients. Radiology 2018;289(03):816-830

12 Roskams T, Kojiro M. Pathology of early hepatocellular carcinoma: conventional and molecular diagnosis. Semin Liver Dis 2010;30 (01):17-25

13 Kojiro M, Roskams T. Early hepatocellular carcinoma and dysplastic nodules. Semin Liver Dis 2005;25(02):133-142

14 Khalili K, Kim TK, Jang H-J, et al. Optimization of imaging diagnosis of $1-2 \mathrm{~cm}$ hepatocellular carcinoma: an analysis of diagnostic performance and resource utilization. J Hepatol 2011;54(04):723-728

15 Darnell A, Forner A, Rimola J, et al. Liver imaging reporting and data system with MR imaging: evaluation in nodules $20 \mathrm{~mm}$ or smaller detected in cirrhosis at screening US. Radiology 2015;275 (03):698-707

16 Aubé C, Oberti F, Lonjon J, et al; CHIC Group. EASL and AASLD recommendations for the diagnosis of HCC to the test of daily practice. Liver Int 2017;37(10):1515-1525

17 van den Bos IC, Hussain SM, Dwarkasing RS, et al. MR imaging of hepatocellular carcinoma: relationship between lesion size and imaging findings, including signal intensity and dynamic enhancement patterns. J Magn Reson Imaging 2007;26(06):1548-1555

18 Rosenkrantz AB, Lee L, Matza BW, Kim S. Infiltrative hepatocellular carcinoma: comparison of MRI sequences for lesion conspicuity. Clin Radiol 2012;67(12):e105-e111

19 Barr DC, Hussain HK. MR imaging in cirrhosis and hepatocellular carcinoma. Magn Reson Imaging Clin N Am 2014;22(03):315-335

20 Brunt E, Aishima S, Clavien PA, et al. cHCC-CCA: consensus terminology for primary liver carcinomas with both hepatocytic and cholangiocytic differentiation. Hepatology 2018;68(01): 113-126

21 Taouli B, Koh D-MK. Diffusion-weighted MR imaging of the liver. Radiology 2010;254(01):47-66

$22 \mathrm{Kim}$ JE, Kim SH, Lee SJ, Rhim H. Hypervascular hepatocellular carcinoma $1 \mathrm{~cm}$ or smaller in patients with chronic liver disease: characterization with gadoxetic acid-enhanced MRI that includes diffusion-weighted imaging. AJR Am J Roentgenol 2011;196(06): W758-65

23 Piana G, Trinquart L, Meskine N, Barrau V, Beers BV, Vilgrain V. New MR imaging criteria with a diffusion-weighted sequence for the diagnosis of hepatocellular carcinoma in chronic liver diseases. J Hepatol 2011;55(01):126-132

24 Park MS, Kim S, Patel J, et al. Hepatocellular carcinoma: detection with diffusion-weighted versus contrast-enhanced magnetic resonance imaging in pretransplant patients. Hepatology 2012;56 (01):140-148

25 Vandecaveye V, De Keyzer F, Verslype C, et al. Diffusion-weighted MRI provides additional value to conventional dynamic contrastenhanced MRI for detection of hepatocellular carcinoma. Eur Radiol 2009;19(10):2456-2466

26 Choi SH, Byun JH, Lim Y-S, et al. Diagnostic criteria for hepatocellular carcinoma $\leq 3 \mathrm{~cm}$ with hepatocyte-specific contrast-enhanced magnetic resonance imaging. J Hepatol 2016;64(05): 1099-1107

27 Parikh T, Drew SJ, Lee VS, et al. Focal liver lesion detection and characterization with diffusion-weighted MR imaging: comparison with standard breath-hold T2-weighted imaging. Radiology 2008;246(03):812-822
28 Muhi A, Ichikawa T, Motosugi U, et al. High-b-value diffusionweighted MR imaging of hepatocellular lesions: estimation of grade of malignancy of hepatocellular carcinoma. J Magn Reson Imaging 2009;30(05):1005-1011

29 Xu PJ, Yan FH, Wang JH, Shan Y, Ji Y, Chen CZ. Contribution of diffusion-weighted magnetic resonance imaging in the characterization of hepatocellular carcinomas and dysplastic nodules in cirrhotic liver. J Comput Assist Tomogr 2010;34(04):506-512

30 Nasu K, Kuroki Y, Tsukamoto T, Nakajima H, Mori K, Minami M. Diffusion-weighted imaging of surgically resected hepatocellular carcinoma: imaging characteristics and relationship among signal intensity, apparent diffusion coefficient, and histopathologic grade. AJR Am J Roentgenol 2009;193(02):438-444

31 Kim AY, Kim YK, Lee MW, et al. Detection of hepatocellular carcinoma in gadoxetic acid-enhanced MRI and diffusion-weighted MRI with respect to the severity of liver cirrhosis. Acta Radiol 2012;53(08):830-838

32 Zhu SC, Liu YH, Wei Y, et al. Intravoxel incoherent motion diffusion-weighted magnetic resonance imaging for predicting histological grade of hepatocellular carcinoma: comparison with conventional diffusion-weighted imaging. World J Gastroenterol 2018;24(08):929-940

33 Wei Y, Gao F, Wang M, et al. Intravoxel incoherent motion diffusion-weighted imaging for assessment of histologic grade of hepatocellular carcinoma: comparison of three methods for positioning region of interest. Eur Radiol 2019;29(02): 535-544

34 Van Beers BE, Pastor CM, Hussain HK. Primovist, Eovist: what to expect? J Hepatol 2012;57(02):421-429

35 Kitao A, Matsui O, Yoneda N, et al. The uptake transporter OATP8 expression decreases during multistep hepatocarcinogenesis: correlation with gadoxetic acid enhanced MR imaging. Eur Radiol 2011;21(10):2056-2066

36 Tsuboyama T, Onishi H, Kim T, et al. Hepatocellular carcinoma: hepatocyte-selective enhancement at gadoxetic acid-enhanced MR imaging-correlation with expression of sinusoidal and canalicular transporters and bile accumulation. Radiology 2010;255 (03):824-833

37 Sano K, Ichikawa T, Motosugi U, et al. Imaging study of early hepatocellular carcinoma: usefulness of gadoxetic acid-enhanced MR imaging. Radiology 2011;261(03):834-844

38 Choi SH, Kim KW, Lee JY, Kim K, Park SH. Diffusion-weighted magnetic resonance enterography for evaluating bowel inflammation in Crohn's disease: a systematic. Inflamm Bowel Dis 2016; 22(03):669-679

39 Lee SA, Lee $\mathrm{CH}$, Jung WY, et al. Paradoxical high signal intensity of hepatocellular carcinoma in the hepatobiliary phase of Gd-EOBDTPA enhanced MRI: initial experience. Magn Reson Imaging 2011;29(01):83-90

40 Choi JY, Kim MJ, Park YN, et al. Gadoxetate disodium-enhanced hepatobiliary phase MRI of hepatocellular carcinoma: correlation with histological characteristics. AJR Am J Roentgenol 2011;197 (02):399-405

41 Kitao A, Zen Y, Matsui O, et al. Hepatocellular carcinoma: signal intensity at gadoxetic acid-enhanced MR Imaging-correlation with molecular transporters and histopathologic features. Radiology 2010;256(03):817-826

42 Fujita N, Nishie A, Kubo Y, et al. Hepatocellular carcinoma: clinical significance of signal heterogeneity in the hepatobiliary phase of gadoxetic acid-enhanced MR imaging. Eur Radiol 2015;25(01): 211-220

43 Motosugi U, Ichikawa $\mathrm{T}$, Sou $\mathrm{H}$, et al. Liver parenchymal enhancement of hepatocyte-phase images in Gd-EOB-DTPAenhanced MR imaging: which biological markers of the liver function affect the enhancement? J Magn Reson Imaging 2009; 30(05):1042-1046

44 Okada M, Murakami T, Kuwatsuru R, et al. Biochemical and clinical predictive approach and time point analysis of 
hepatobiliary phase liver enhancement on Gd-EOB-DTPA-enhanced MR images: a multicenter study. Radiology 2016;281 (02):474-483

45 Kambadakone AR, Sahani DV. Body perfusion CT: technique, clinical applications, and advances. Radiol Clin North Am 2009; 47(01):161-178

46 Taouli B, Johnson RS, Hajdu CH, et al. Hepatocellular carcinoma: perfusion quantification with dynamic contrast-enhanced MRI. AJR Am J Roentgenol 2013;201(04):795-800

47 Sahani DV, Holalkere NS, Mueller PR, Zhu AX. Advanced hepatocellular carcinoma: CT perfusion of liver and tumor tissue-initial experience. Radiology 2007;243(03):736-743

48 Chen J, Chen C, Xia C, et al. Quantitative free-breathing dynamic contrast-enhanced MRI in hepatocellular carcinoma using gadoxetic acid: correlations with Ki67 proliferation status, histological grades, and microvascular density. Abdom Radiol (NY) 2018;43 (06):1393-1403

49 Eisenhauer EA, Therasse P, Bogaerts J, et al. New response evaluation criteria in solid tumours: revised RECIST guideline (version 1.1). Eur J Cancer 2009;45(02):228-247

50 Lencioni R, Llovet JM. Modified RECIST (mRECIST) assessment for hepatocellular carcinoma. Semin Liver Dis 2010;30(01):52-60

51 Lencioni R, Montal R, Torres F, et al. Objective response by mRECIST as a predictor and potential surrogate end-point of overall survival in advanced HCC. J Hepatol 2017;66(06):1166-1172

52 Ronot M, Bouattour M, Wassermann J, et al. Alternative response criteria (Choi, European association for the study of the liver, and modified Response Evaluation Criteria in Solid Tumors [RECIST]) Versus RECIST 1.1 in patients with advanced hepatocellular carcinoma treated with sorafenib. Oncologist 2014;19(04):394-402

53 Gavanier M, Ayav A, Sellal C, et al. CT imaging findings in patients with advanced hepatocellular carcinoma treated with sorafenib: alternative response criteria (Choi, European Association for the Study of the Liver, and modified Response Evaluation Criteria in Solid Tumor (mRECIST)) versus RECIST 1.1. Eur J Radiol 2016;85 (01):103-112
54 Reig M, Rimola J, Torres F, et al. Postprogression survival of patients with advanced hepatocellular carcinoma: rationale for second-line trial design. Hepatology 2013;58(06):2023-2031

55 Iavarone M, Cabibbo G, Biolato M, et al. Predictors of survival in patients with advanced hepatocellular carcinoma who permanently discontinued sorafenib. Hepatology 2015;62(03):784-791

56 Nishino M, Hatabu H, Hodi FS. Imaging of cancer immunotherapy: current approaches and future directions. Radiology 2019;290 (01):9-22

57 Seymour L, Bogaerts J, Perrone A, et al; RECIST working group. iRECIST: guidelines for response criteria for use in trials testing immunotherapeutics. Lancet Oncol 2017;18(03):e143-e152

$58 \mathrm{Kim}$ SY, An J, Lim Y-S, et al. MRI with liver-specific contrast for surveillance of patients with cirrhosis at high risk of hepatocellular carcinoma. JAMA Oncol 2017;3(04):456-463

59 Kim HL, An J, Park JA, Park SH, Lim YS, Lee EK. Magnetic resonance imaging is cost-effective for hepatocellular carcinoma surveillance in high-risk patients with cirrhosis. Hepatology 2019;69(04):1599-1613

60 Singal A, Volk ML, Waljee A, et al. Meta-analysis: surveillance with ultrasound for early-stage hepatocellular carcinoma in patients with cirrhosis. Aliment Pharmacol Ther 2009;30(01):37-47

61 Zhou W, Zhang L, Wang K, et al. Malignancy characterization of hepatocellular carcinomas based on texture analysis of contrast-enhanced MR images. J Magn Reson Imaging 2017;45 (05):1476-1484

62 Rosenkrantz ABPN, Pinnamaneni N, Kierans AS, Ream JM. Hypovascular hepatic nodules at gadoxetic acid-enhanced MRI: wholelesion hepatobiliary phase histogram metrics for prediction of progression to arterial-enhancing hepatocellular carcinoma. Abdom Radiol (NY) 2016;41(01):63-70

63 Kim J, Choi SJ, Lee SH, Lee HY, Park H. Predicting survival using pretreatment CT for patients with hepatocellular carcinoma treated with transarterial chemoembolization: comparison of models using radiomics. AJR Am J Roentgenol 2018;211(05):1026-1034

64 Gillies RJ, Kinahan PE, Hricak H. Radiomics: images are more than pictures, they are data. Radiology 2016;278(02):563-577 